\title{
Support vector machine based feature extraction for gender recognition from objects using lasso classifier
}

\author{
Damodara Krishna Kishore Galla ${ }^{{ }^{*}}$, Babu Reddy Mukamalla ${ }^{1}$ and Rama Prakasha Reddy Chegireddy ${ }^{2}$
}

\author{
*Correspondence: \\ kishore.galla1@gmail.com \\ ${ }^{1}$ Computer Science, Krishna \\ University, Machilipatnam, \\ A.P, India \\ Full list of author information \\ is available at the end of the \\ article
}

\begin{abstract}
Object detection and gender recognition were two different categories to be classified in a single section is a complicated task and this approach helps in supporting the blind people for an artificial vision. In this paper, our method to the betters vision sensation of blind persons by conversion of visualized data to audio data. Therefore this artificial intelligence model helps in detecting the objects as well as human face recognition with gender classification based on face recognition approach. This model processed with feature extraction and classification models. The feature extraction was comprised with multi scale-invariant feature transform (MSIFT), with feature optimization with support vector machine algorithm then classified using LASSO classifier. For better performance identification, three different classification models were implemented and tested too. Feature selection helps in making tests early to detect the objects and recognizing human actions using image processing approach. This approach can be applied for both offline and online modes. But in this scenario, an offline mode was implemented and was tested with a combination of different databases. For this process of classification ridge regression (RR), elastic net (EN), lasso regression (LR) and LASSO regression were implemented. The final classification results with accuracy are as follows for RR-89.6\%, EN-93.5\%, LR-93.2\% and proposed approach (LRGS) with 98.4\% accurate detection rate with prediction name of classes.
\end{abstract}

Keywords: Support vector machine, Ridge regression, Eleastinet, Logistic regression, LRGS, Gender classification

\section{Introduction}

Sex or gender is a feature that helps to identify a person initially. Gender-based separation among humans is classified into two: male and female. Specific gender groups might also show off diverse propensities. For instance, younger guys and young girls may like toys; human beings may incline a variety of clothes varying in colours and styles. In enterprise understanding, to know the gender (and age) of the customers may also assist business chiefs to publicize their objects as indicated by using numerous purchaser groups and accumulate sizeable statistic facts about the clients, for instance, the number of women entering during a retail region or a shopping centre inside a given period. In picture healing, gender (or age) can be a treasured semantic 
concept for photo association and search, as coming across men or women in specific scenes. In social collaborations or exercises, guys and ladies can also perform contrastingly and have numerous jobs. In this manner perceiving the gender of a person has extensive variations. People can see the gender of each other. It has been dynamic research in brain science to reflect on consideration on the effect of gender on people [1-3]. In brain technology about, the development is usually the face photographs for gender popularity. The visible computational investigation for gender characterization or acknowledgement, a large portion of the current works applied face pictures. For example, see [4-8].

As of past due, some methodologies, for example, $[9,10]$, established that human bodies must use be for gender grouping. Victories display on available dataset images wherein a bodily drawn box containing someone walking utilized all round for frame records extraction. The upsides of using body over face pictures for gender acknowledgement include the accompanying list.

(1) Image Resolution. When the face locale has a shared dream or is motion obscured, the face highlights in all likelihood won't be usable for gender order. Be that as it can, the self-belief may also at gift help isolate guys from ladies.

(2) Viewpoint Change. At the factor when the head present is altogether one of a kind from frontal perspectives, the face-primarily based on gender acknowledgement may additionally have poor execution or even can not be appropriate. Notwithstanding, self-perception can at gift use. Indeed, even the returned angle on the frame can be used for gender acknowledgement $[9,10]$.

(3) Acquisition Distance. At the factor when the camera is some distance away from the person, the face photograph won't supply good enough records to gender segregation. Be that as it could, self-belief may additionally even now be usable.

(4) Occlusion. At the point, when the facial part is blocked, the face image probable might not be applied to do away with gender statistics. Be that as it can, the selfnotion is as but useful, in any event, while a person features are impeded. Be that as it could, in the ones spearheading ways to deal with sex acknowledgement from the body, as an instance, $[9,10]$, merely the upstanding self-perceptions with the complete frame look explored. The information customarily applied are individual strolling images, as an instance, the MIT passer-by database [11], can be named, which is an interactive database for walker identity. One pressing necessity is that the whole body in upstanding shows up in each picture, as regarded in Fig. 1, and in this way, there is no compelling motive to pressure over to adjust the self-perceptions in coordination. Highlights can be extricated from the entire self-perceptions and used to put together a classifier.

The organization of the paper is as follows, in Literature survey section gives various machine learning schemes applied for object detection and face-based gender recognition were identified. In "Proposed methods" section, SVM based feature extraction in combination with MSIFT (Multi-Scale Invariant Feature Transform) demonstrated along with LASSO based regression classification analysis, in "Results and discussion" section various dataset based results were plotted along with simulation setup. 


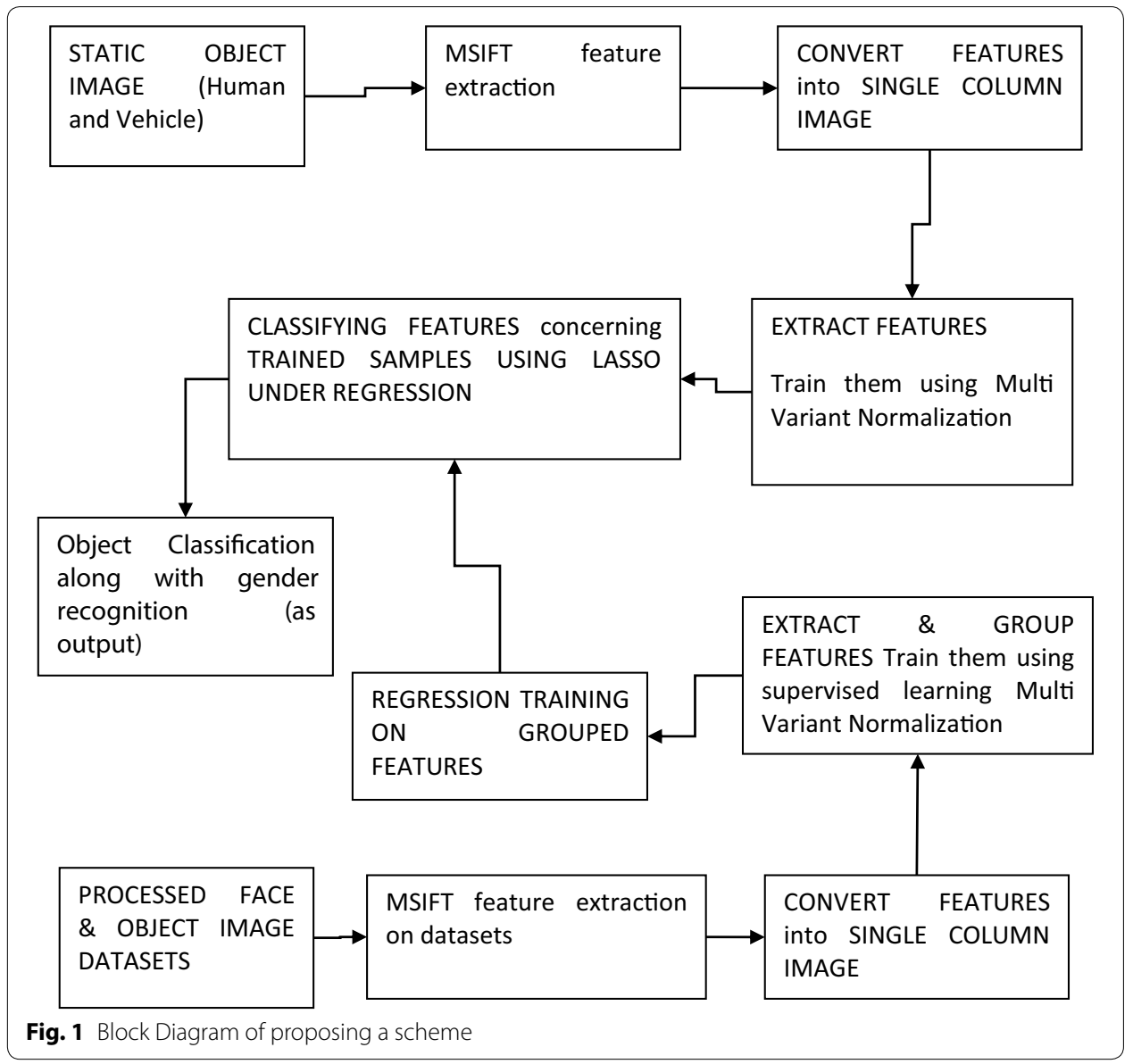

"Conclusion" section concludes the paper with a brief note on how our research paper finds a solution with performance metrics.

\section{Literature survey}

One of the simple issues with any photo making prepared or PC vision undertaking is the clear up of a becoming language for addressing the realities. Face association carries the extraction of a collection of parameters or estimations that permit portraying of the variations between human beings, instead of the sorts amongst snapshots of individuals.

This method is authentic notably after the photographs have been coded with the component of the dismissal of non-facial variations as the internal man or woman, regardless need to be banned. A regularly carried out method for characterization is Support Vector Machine (SVM) [1, 12]; those tune into the ideal hyper-flying system for keeping apart several getting ready by way of the method for confining the peril of mis-association. Recognization method is regularization or subject-based strategy. SVMs have in recent times been used for face grouping [10,13], displaying dependably better. These compositions grew to become out to be maximum of the way maintained through the Ministry of Education, Culture, Sports, Science and Technology Grant-in-Aid for Scientific Research (B) 15300076. The makers are thankful to the Softopia Japan Foundation for the HOIP dataset. Prevalence execution than famous 
nearest neighbour strategies. Facial sex plan has, also, been taken into consideration $[3,6,9,11]$. Likewise, a non-instantly SVM based honestly classifier has appeared to make through and via higher outcomes than particular frameworks [14].

The sizeable separation between sexual orientations changed into approval to be strikingly twisted, and this comes to be the pinnacle gone to utilize the help of vector attitude. Regardless, none of those structures chooses to become, robust estimations at which point to organize the countenances. In this paper, a machine for showing up highlight the determination-making use of SVMs delineated wherein the improvement occurs in the parameter location.

Using the board model throughout the work, a pitiful combination of features made with a considerable scope of the classifier's parameter is assessed to be 0 . The capabilities are greater vigorous than SVM estimations and are efficaciously among the considered. This tool has been named Lasso regression [15], or dynamic [1] or purpose intrigue [4] characterization.

When acting characteristic devotion, it's miles simple to realize the trustworthiness of the element choice method. This approach can do with the aid of discovering the most elements with bunches of complicated insufficient classifiers. In this paper, a different approach for finding the entire relationship of small SVMs is portrayed, which produces internationally perfect classifiers for the development of linear programming sub-problems.

It is then suggested to ap-contract Automatic Relevance Detection (ARD) [16] and choose alone rendition it is enhancing the probability of the practice statistics explained over the various stages of scantiness. At the same time, it is the far some distance average that numerous portrayals and an ever-growing number of versatile amounts supply the advanced arrangement exactness.

The simple thought system in this paper is to depict how the individual who wants, and between congruity of confirmation to bring about talented highlights works of art and smash down their duties in a prime dimension between containing a choice problem. All these research papers suggested object classification or gender recognition statements. But on a combination, these were not made especially object (Vehicle) detection and human face based gender recognition combination wasn't made enough and had no perfect pretrained dataset available. For this purpose then this made us clear to develop a dataset with combination of both objects and human face recognition system under single classification approach which will help the blind one to identify the surroundings effectively, but this wasn't found in any of the systems early developed.

Most of the approaches mentioned in this section were focused on gender recognition with the help of various classifiers, and most of the approaches were applied on nominal databases.

The significant contributions of all other researchers were stopped with just detecting the gender with 2 class identification approach. However, here in this research article, a combination of 13 classes which supports in identifying the objects and human faces will separate in them, and the gender will be identified. This approach made other researchers think on blind vision people problem solution tracing. 


\section{Proposed methods}

In this paper, proposing scheme comprised with Multi scale-invariant feature extraction and then these features converted into image frame then these get normalized by using multi-variant normalization. This the feature extraction process followed by the training process. Then these two gets classified by using MSVM, GSVM, LASSO, Logistic Regression based LASSO, ELASTIC NET, RIDGE and compared these results in the results section of the paper. The entire process was comprised and showcased in block diagram Fig. 1.

Over comparing with all existing approaches, i.e., object recognition or face recognition system deployment feature selection and grouping them with the same procedure isn't a part of existing approaches. But in our proposing scheme, the combinational feature combined and worked on various algorithms.

The database generation for objects and face recognition is a complicated term but in our approach with advanced MSIFT and clustering of the feature made this possible.

\section{Feature extraction}

\section{Classification}

Table 1 represents the different feature extraction process that was implemented along with our proposing approach to verify which feature extraction will result in the high accuracy rate for classification. In Table 1, the results column helps in.

Selecting the best approach for feature selection process applied by the support vector machine.

Logistic regression a modelling method for estimating the $\theta(\boldsymbol{X})=P(Y=1 \mid \boldsymbol{X})$, and hence $P(Y=0 \mid X)=1-P(Y=1 \mid X)=1-\theta(X)$ As well. Thus it can be used as a classification method for binary classification problems, i.e. the nominal response has two levels, generically "Yes/Success $(Y=1)$ " and "No/Failure $(Y=0)$ ".

Our general classification rule based on these estimated probabilities is given by, If $\widehat{\theta}(\boldsymbol{X})=P(Y=1 \mid X) \geq p$ then classifies $Y$ as being from class 1, i.e. "Yes" or "Success". If $\widehat{\theta}(\boldsymbol{X})=P(Y=1 \mid \boldsymbol{X})<p$ then classify $Y$ as being from class 0, i.e. "No" or "Failure".

$p=0.50$ Makes the most sense from a logical standpoint, but we could certainly use other values. Also, we can rank observations based on these estimated probabilities to find most likely observations/cases where $Y=1$ (see the "Discussion" of Lift in Section 12).

The probabilities are estimated using a generalized linear model (GLM) for the natural $\log$ of the odds for "success", which is called the logistic $(L)$. The logistic regression model is given by,

$$
L=\ln \left(\frac{\theta(\boldsymbol{X})}{1-\theta(\boldsymbol{X})}\right)=\beta_{o}+\beta_{1} U_{1}+\beta_{2} U_{2}+\ldots+\beta_{k-1} U_{k-1}
$$

As was the case with the general OLS model, the terms $\left(U_{j}^{\prime} s\right)$ are all functions of the predictors $\left(X_{1}, X_{2}, \ldots, X_{p}\right)$. Once we have obtained estimates of the model parameters $\left(\widehat{\beta}_{j}^{\prime} s\right)$, and hence the estimated logit $\widehat{L}$, we can estimate the probability of "Yes/Success" as, 
Table 1 Different feature extraction algorithms on FERET data sets

\begin{tabular}{|c|c|c|c|c|}
\hline S. No & $\begin{array}{l}\text { Feature } \\
\text { Extractor }\end{array}$ & Formula & Remark & Result analysis \\
\hline 1 & $\begin{array}{c}\text { Gabor PCA } \\
\text { [19] }\end{array}$ & $\begin{array}{l}G P=\underset{\operatorname{argmax}}{\operatorname{areal}(\mid * \psi)^{2}+} \\
\left.\quad I m g(\mid * \psi)^{2}\right) * \lambda\end{array}$ & $\begin{array}{l}\text { Extracted real and imagi- } \\
\text { nary features of Gabor } \\
\text { (G) were optimized by } \\
\text { PCA (P) }\end{array}$ & $\begin{array}{l}\text { Extracts edges from } \\
\text { the edges by dif- } \\
\text { ferent frequency } \\
\text { scaling components } \\
\text { concerning PCA } \\
\text { provides a recogni- } \\
\text { tion rate of } 89.4 \%\end{array}$ \\
\hline 2 & $\begin{array}{l}\text { Gabor } \\
\text { MEAN } \\
\text { PCA [19] }\end{array}$ & $\begin{array}{l}G P=\underset{\operatorname{argmax}}{ }\left(\sum\left(\operatorname{Real}(\mid * \psi)^{2}+\right.\right. \\
\left.\left.\quad I m g(\mid * \psi)^{2}\right)\right) * \lambda\end{array}$ & $\begin{array}{l}\text { Here mean of Gabor filter } \\
\text { were subjected to PCA } \\
\text { optimization }\end{array}$ & $\begin{array}{l}\text { All the extracted } \\
\text { edges applied by } \\
\text { mean and provide a } \\
\text { recognition rate of } \\
92.7 \%\end{array}$ \\
\hline 3 & HOG [8] & $H=\frac{v}{\sqrt{v_{2}^{2}+e^{2}}}$ & $\begin{array}{l}\text { This theory will perform } \\
\text { local feature extraction } \\
\text { and normalization. }\end{array}$ & $\begin{array}{l}\text { All the block-wise } \\
\text { extracted histogram } \\
\text { features will result in } \\
\text { an under-recogni- } \\
\text { tion rate of } 83.9 \%\end{array}$ \\
\hline 4 & SIFT [12] & $\begin{array}{l}\text { Scale } \\
D(x, y, \sigma)=L\left(x, y, K_{i} \sigma\right)-L\left(x, y, K_{j} \sigma\right) \\
\text { Position: } \\
D(x)=D+\frac{\partial D^{T}}{\partial x} x+\frac{1}{2} x^{T} \frac{\partial^{2} D}{\partial x^{2}} x \\
\text { Orientation: } \\
m(x, y)= \\
\sqrt{(L(x+1)-L(x-1))^{2}+(L(y+1)-L(y-1))^{2}} \\
\theta(x, y)=\operatorname{atan} 2(L(y+1)-L(y-1), \\
\quad L(x+1)-L(x-1))\end{array}$ & $\begin{array}{l}\text { One descriptor base four } \\
\text { features will be extracted } \\
\text { from the SIFT algorithm, } \\
\text { and point descriptor of } \\
\text { the best form will help } \\
\text { exact identification of } \\
\text { objection. Even the scale } \\
\text { of the object varied yet } \\
\text { will recognize it. }\end{array}$ & $\begin{array}{l}\text { As per remarks, it } \\
\text { compares the } \\
\text { object with point } \\
\text { key descriptors } \\
\text { and results with an } \\
\text { accuracy of } 92.7 \% \\
\text { for recognition }\end{array}$ \\
\hline 5 & $\begin{array}{l}\text { MSIFT- } \\
\text { Proposing } \\
\end{array}$ & $\begin{array}{l}\text { Scale } \\
D(x, y, \sigma)=\left(L\left(x, y, K_{i} \sigma\right)-L\left(x, y, K_{j} \sigma\right)\right) * \Psi \\
\text { Position: } \\
D(x)=\left(D+\frac{\partial D^{T}}{\partial x} x+\frac{1}{2} x^{T} \frac{\partial^{2} D}{\partial x^{2}} x\right) * \Psi \\
\text { Orientation: } \\
m(x, y)= \\
\quad \sqrt{(L(x+1)-L(x-1))^{2}+(L(y+1)-L(y-1))^{2}} \\
\theta(x, y)=\operatorname{atan} 2(L(y+1)-L(y-1), \\
\quad L(x+1)-L(x-1))\end{array}$ & \multicolumn{2}{|c|}{$\begin{array}{ll}\text { The image is split into mul- } & \text { The modified SIFT } \\
\text { tiple frequencies com- } & \text { results in most } \\
\text { ponents and extracted } & \text { format and results in } \\
\text { sift features from them } & \text { the best recognition } \\
\text { will result in effective } & \text { rate of } 98.3 \% \text { in this } \\
\text { detection even in images } & \text { proposing approach } \\
2 & \text { of different sizes. }\end{array}$} \\
\hline
\end{tabular}

$$
\widehat{\theta}(\boldsymbol{X})=\frac{e^{\widehat{L}}}{1+e^{\widehat{L}}}=\frac{1}{1+e^{-\widehat{L}}}
$$

Model selection (e.g. stepwise methods) and cross-validation (if the goal is accurate prediction) are essential elements of the model building process in logistic regression. Term creation, for example, power transformations and interactions, is less straight forward for these models but can be crucial in developing a "good" model for a given situation. On the next page, some guidelines for term creation in logistic regression given.

Our proposing approach comprised of three stages as earlier mentioned, namely features extraction, feature optimization and classification.

With the help of Table 1 feature extraction made possible, and feature optimization was done with the help SVM classifier and algorithm is given below in Algorithm 1. 


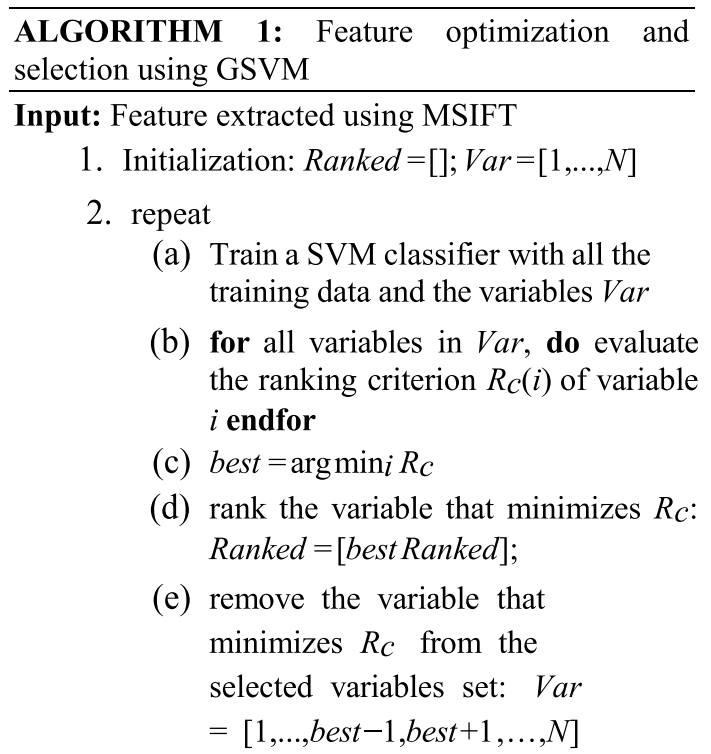

3. until Var is not empty

Output: Optimized reduced features.

The reduced features were subjected to lasso classification models. This feature optimization reduces the test time response and helps in obtaining pleasing experimental results for the datasets combination we considered. Then the classification procedure will be carried out with the help of the following Algorithm 2 with the help of Table 2.

Table 2 Univariate Considerations [15]

\begin{tabular}{|c|c|}
\hline $\begin{array}{l}f(x \mid y) \text {-conditional distribution } \\
x \text { gave as } y \text { which is } 0 \text { or } 1 .\end{array}$ & Suggested model terms \\
\hline $\begin{array}{l}\text { The standard, common variance } \\
\text { i.e. } \operatorname{Var}\left(x_{j} \mid y=0\right)=\operatorname{Var}\left(x_{j} \mid y=1\right)\end{array}$ & $\begin{array}{l}X_{j} \text {, i.e. the predictor itself } \\
\text { These values imply that i } X_{j} \text { t is NOT customarily } \\
\text { distributed we might consider transforming } X_{j} \text { to } \\
\text { approx. Normality. }\end{array}$ \\
\hline $\begin{array}{l}\text { Normal, unequal variances } \\
\text { i.e. } \operatorname{Var}\left(x_{j} \mid y=0\right) \neq \operatorname{Var}\left(x_{j} \mid y=1\right)\end{array}$ & $X_{j}$ and $X_{j}^{2}$ \\
\hline Skewed right & $\begin{array}{l}X_{j} \text { and } \log _{2}\left(X_{j}\right) \\
\text { Log base } 2 \text { is more comfortable to interpret }\end{array}$ \\
\hline$x \in[0,1]$ & $\log _{2}\left(X_{j}\right)$ and $\log _{2}\left(1-X_{j}\right)$ \\
\hline$X_{j} \sim$ Poisson i.e. $X_{j}$ is a count & $X_{j}$, i.e. the predictor itself \\
\hline
\end{tabular}




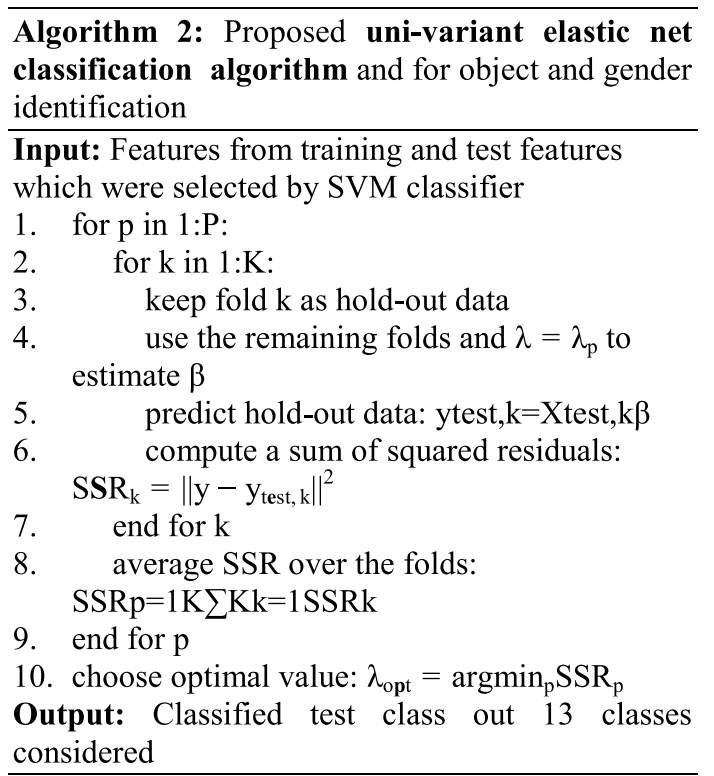

Here $\mathrm{p}$ indicates the test samples, $\lambda$ indicated the train samples, $\mathrm{k}$ is the size of total features, $\beta$ is sample match count, $y \& y_{\text {test }}$ are labelling match condition with respect residuals.

As the residual identification for the optimized features made the classification rate to improve and results in obtaining the best performance metric values. These two algorithms implementation made the test results more effective in combination with different feature extraction algorithms were tested and mentioned the database based results in the results section. This approach made an error rate to suppressed and enhances the accuracy rate of the system, along with the minimization of testing time.

\section{Multivariate considerations}

When considering multiple continuous predictors simultaneously, we look at multivariate normality.

$$
f(\underset{\sim}{x} \mid y) \sim M V N\left(\mu_{y=k}, \Sigma\right)
$$

then use the x's themselves

$$
f(\underset{\sim}{x} \mid y) \sim \operatorname{MVN}\left(\mu_{y=k}, \Sigma_{y=k}\right)
$$

then include $X_{j}^{2}$ 's and $X_{i} X_{j}$ terms

For example in the two predictor case $(p=2) x_{1} x_{2}$ is needed if $E\left(x_{1} \mid x_{2}\right)=\beta_{o}+\beta_{1, y=k} x_{2}$

Moreover, if the variances are different for the $x_{i}$ across levels of $y$ then, we add $x_{i}^{2}$ terms as well. A scatterplot matrix with the colour of the points coded by the levels of the response $Y$ is a useful tool for visualizing which situation is appropriate for our classification problem. 


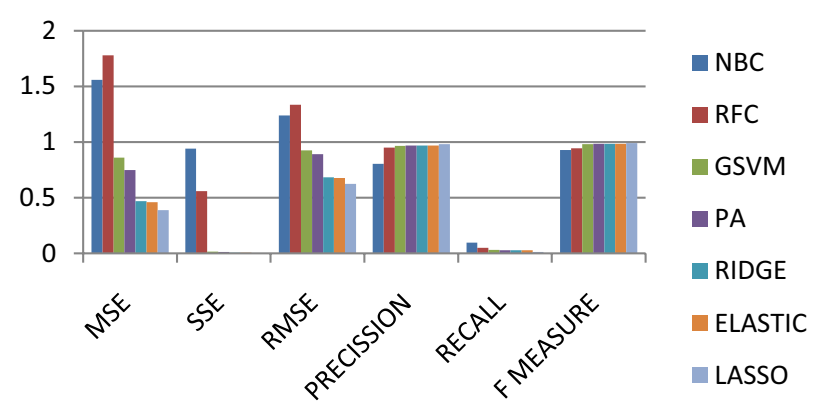

Fig. 2 Performance metrics for different classifiers under FG-NET database

In cases where this instability in the predicted probabilities happens (as in the previous example), ridge, LASSO, and Elastic Net logistic regression are good options. These are also good options when one has a "wide data" problem where $n<p$ or when $\mathrm{p}$ is large and also when you have some highly correlated predictors. For logistic regression, the regularised logistic models using the ridge and Lasso given below.

Ridge Logistic:

$$
\ln \left(\frac{\theta(\boldsymbol{x})}{1-\theta(\boldsymbol{x})}\right)=\beta_{o}+\sum_{j=1}^{k} \beta_{j} u_{j}+\lambda \sum_{j=1}^{k} \beta_{j}^{2}
$$

Lasso Logistic:

$$
\ln \left(\frac{\theta(\boldsymbol{x})}{1-\theta(\boldsymbol{x})}\right)=\eta_{o}+\sum_{j=1}^{k} \beta_{j} u_{j}+\lambda \sum_{j=1}^{k}\left|\beta_{j}\right|
$$

Elastic Net Logistic:

$$
\ln \left(\frac{\theta(\boldsymbol{x})}{1-\theta(\boldsymbol{x})}\right)=\eta_{o}+\sum_{j=1}^{k} \beta_{j} u_{j}+\lambda_{1} \sum_{j=1}^{k}\left|\beta_{j}\right|+\lambda_{2} \sum \beta_{j}^{2}
$$

As mentioned in algorithm 2 Eqs. (3) \& (4) of this section were helps in obtaining the result and minimizing the error rate and then different tests cases were subjected to Eqs. (5) (6) and (7) as kernel function of models for classification and compared using the tabular results in results section

\section{Results and discussion}

For simulation MATLAB (2018b) GUI was used with I3 processor based composition and $8 \mathrm{~GB}$ ram. Initially the GUI was set face recognition system then the system upgraded with MIOTCD vehicle and object database. Then training and classification was performed using the GUI.

\section{Datasets performance metrics with description}

All the graphs from Figs. 2, 3, 4, 5, 6, and 7 were generated with the help of MS word with the values obtained during the simulation in matlab. Here $x$-axis indicates the 


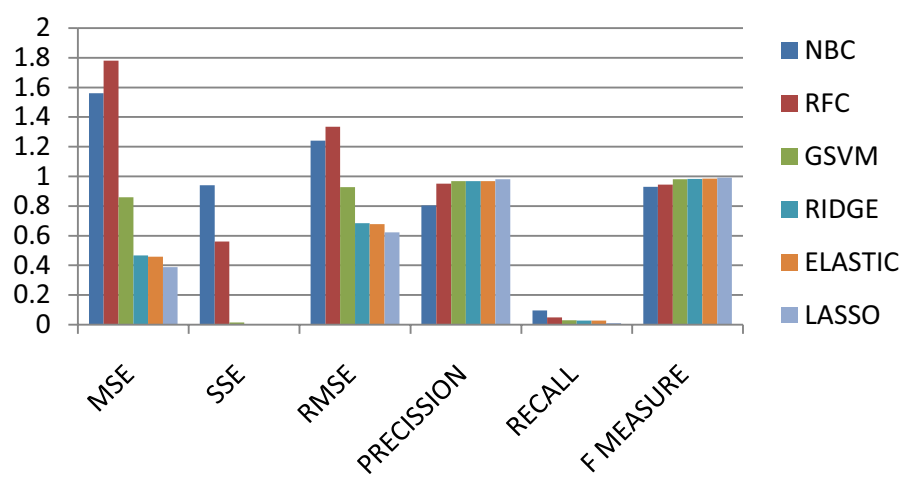

Fig. 3 Performance metrics for different classifiers under ORL database

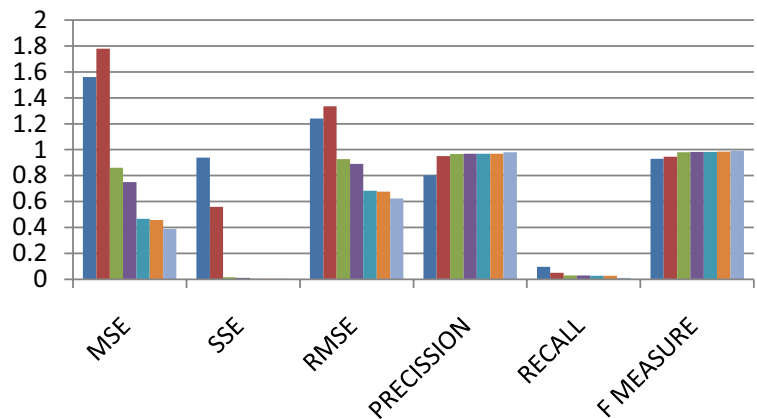

\section{- NBC \\ - RFC \\ - GSVM \\ $\square \mathrm{PA}$ \\ - RIDGE \\ - ELASTIC \\ - LASSO}

Fig. 4 Performance metrics for different classifiers under FERET database

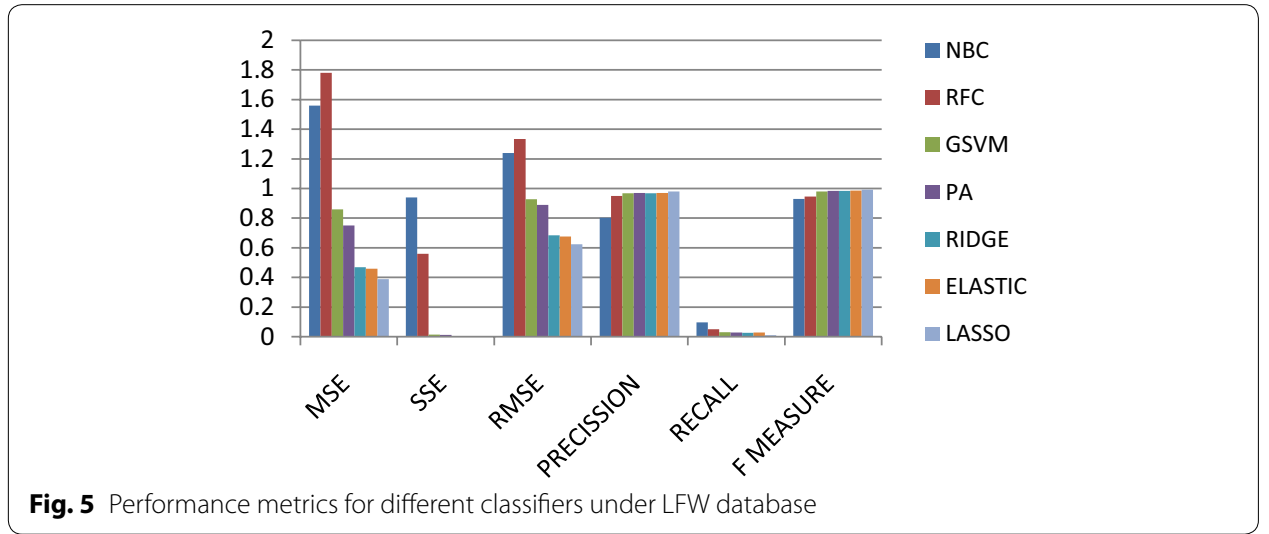

performance metrics and y-axis provides error rate for different datasetes, as the accuracy was high and nearly $98.3 \%$ for all the datasets the performance were so closer and approximately equal to all the datasets.

\section{FG-NET database}

The face and gesture recognition network (FG-NET) database discharged in 2004. The FG-NET database is a freely accessible picture database containing face pictures of 


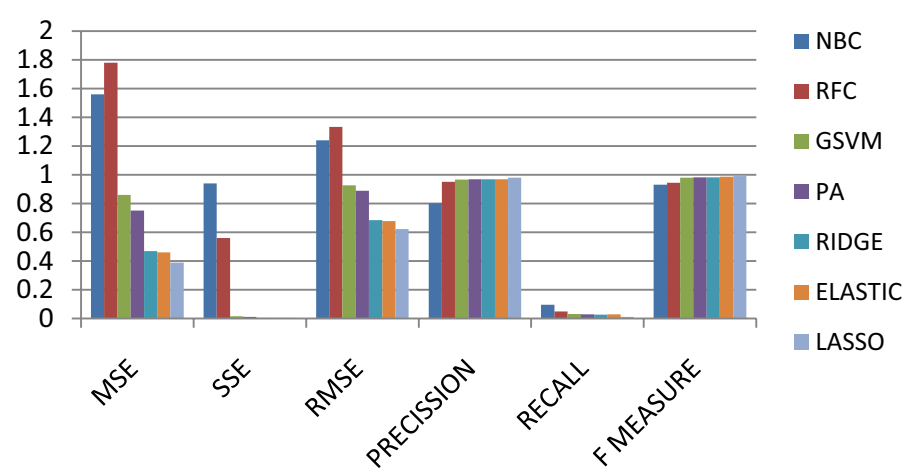

Fig. 6 Performance metrics for different classifiers under PRIVATE database

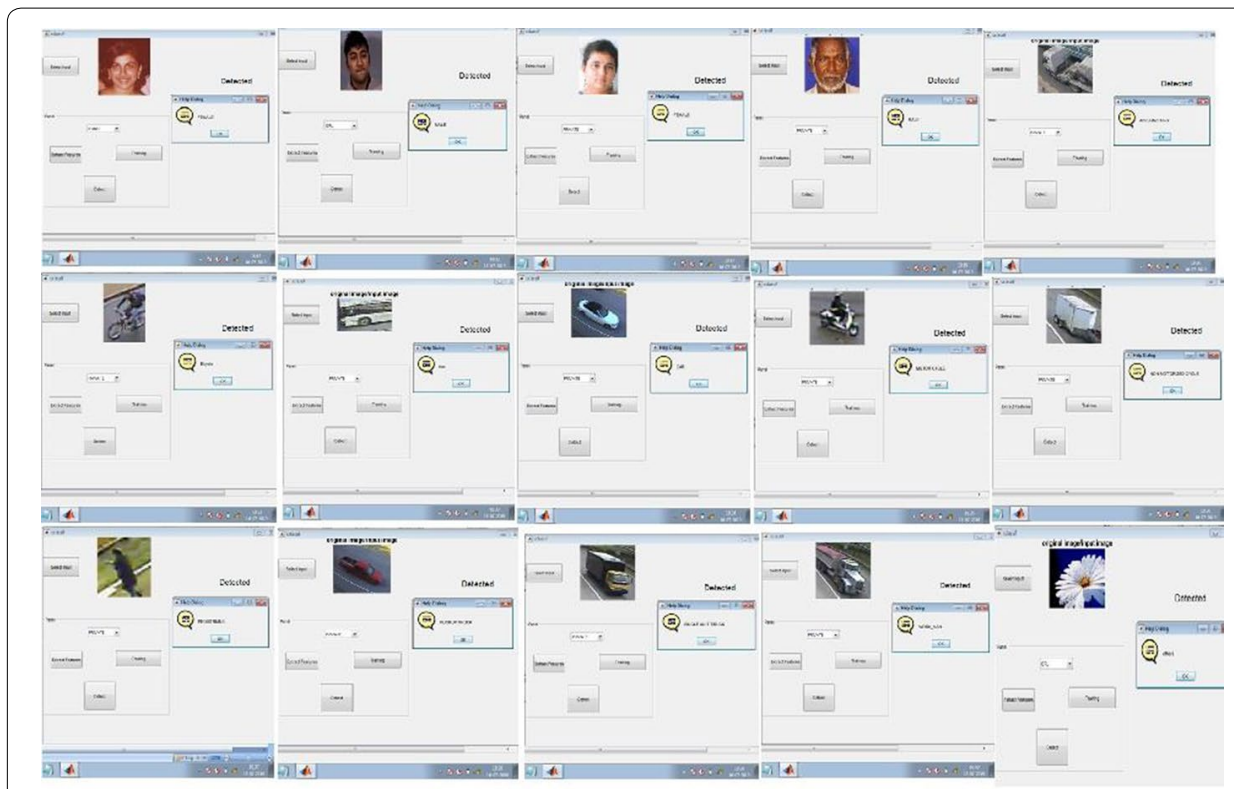

Fig. 7 User interface design for uploading the image to classify objects

several subjects at various ages not just for age estimation for gender based extraction at various ages. The database contains 1002 pictures from 82 distinct subjects with ages extending between infants to 69 years of ages. Be that as it may, ages between zero to 40 years are the most populated in the database.

\section{ORL database}

ORL (Olivetti Research Laboratory) database [17] contains many face images taken between April 1992 and April 1994 at the lab. The database utilized with regards to a face based gender extraction task did in a joint effort with the Speech, Vision and Robotics Group of the Cambridge University Engineering Department. There are ten unique images of every one of 40 particular subjects. For certain subjects, the images were taken on various occasions, differing the lighting, outward appearances and facial subtleties. Every one of the images taken against a dim homogeneous foundation with the subjects 
in an upright, frontal position. Ten various images of every one of 40 particular subjects. For certain subjects, the images were taken on various occasions, fluctuating the lighting, outward appearances.

\section{FERET database}

The FERET database gathered in 15 sessions between August 1993 and July 1996. The database contains 1564 arrangements of pictures for a sum of 14,126 pictures that incorporates 1199 people and 365 copy sets of pictures. A copy set is a moment set of pictures of an individual as of now in the database and typically taken on an alternate day. The Facial Recognition Technology (FERET) database is a dataset utilized for facial based gender extraction framework assessment as a significant aspect of the Face Recognition Technology (FERET) program. The FERET database fills in as a standard database of facial pictures for specialists to use to create different calculations and report results.

\section{LFW database}

(LFW) Labeled Faces in the Wild, a database of face photos intended for examining the issue of the unconstrained face-based gender extraction. The informational collection contains more than 13,000 pictures of appearances gathered from the web. Each face has marked with the name of the individual imagined. One thousand six hundred eighty of the general population imagined having at least two unmistakable photographs in the informational collection.

\section{Private database}

Private database contains various gender orientations and also had various ages of coloured facial images with proper resolution. Human images are accumulated through the camera with quality facial images with $275^{*} 314$ dimensional measurements and also $254 \mathrm{dpi}$ resolutions. The data base comprised with different age group ones, but These images are in JPEG format with reasonable contrast and white balance. The database provided 259 images gathered for face recognition.

\section{Simulation setup}

In this section, the paper describes the experimental setup for different objects based on feature extractions concerning facial and other related images. For simulation MATLAB latest version with minimum $4 \mathrm{~GB}$ RAM and $250 \mathrm{HD}$ for processing multi-label images. Implementation of design for uploading data sets shown in Fig. 7.

Matched and mis-matched class detection is carried out by various algorithms and compared with performance metrics like MSE, RMSE, SSE, PRECISION, RECALL, FMEASURE, ACCURACY and TIME for various datasets. In Fig. 4, to show case the performance of different classification algorithms naive bayes (NBC), Random Forest (RFC), Gaussian SVM (GSVM), Fuzzy C Means Gaussian SVM (FGSVM) [18],

Multi-variant ridge logistic regression (RIDGE), Multi-variant elastic net logistic regression (ELASTIC), Multivariant Lasso logistic regression (LASSO)Here data set size varied based results are tabulated for accuracy and time for various datasets mentioned in the initial stages of results section. 


\section{Performance metrics}

The Metrics used to compare the performance of various algorithms for feature extraction, selection and classification are Mean Square Error, Accuracy, Precision, Recall, Sum of Squared Error, Root Mean Square Error, F measure.

Their formulation is dependent on true positive, true negative, false positive and false negative conditions. These were selected during the classification of the images and were mathematically represented using the below equations.

$$
\begin{aligned}
& \text { Accuracy }=\frac{T P+F N}{T S} \\
& F-\text { Measure }=2 *\left(\frac{1}{\frac{1}{\text { Precision }}+\frac{1}{\text { Recall }}}\right) \\
& \text { Precision }=\frac{T P}{T P+F P} \\
& \text { Recall }=\frac{T P}{T P+F N} \\
& \text { MSE }=\frac{1}{N} \sum_{j=1}^{1}\left(y_{j}-\widetilde{y}_{j}\right)^{2} \\
& \text { SSE }=\sum_{j=1}^{1}\left(y_{j}-\widetilde{y}_{j}\right)^{2} \\
& \text { RMSE }=\sqrt{\frac{1}{N} \sum_{j=1}^{1}\left(y_{j}-\widetilde{y}_{j}\right)^{2}}
\end{aligned}
$$

Figures 3, 4, 5, and 6 resembles the performance metrics for different datasets with effective minimization of error. In all the figures mentioned above $6 \%$ to $8 \%$ effective in F-measure for the proposing scheme similarly RMSE has $3 \%$ change, MSE has $6 \%$ similar to SSE.

In Table 3 classification algorithms based accuracy on FGNET was observed, from Tables 4, 5, 6, 7, and 8 Accuracies were compared with different databases under different classifiers was presented. Outratedly our proposing approach delivers the best accuracy out of all algorithms with a minimum of $3 \%$ best rate.

In Table 9 the above face databases were combined with object databases named MIOTCD and formed a total of 13 classes each section of training images considered 130 samples. For testing of each class 50 samples were considered.

The accuracies were not effective in case of combination for existing and proposing algorithms. In future direction the combination accuracy can be updated. 
Table 3 Accuracy and Time comparison of 3proposing classification algorithms with MSIFT features training based on Multi-variant principle for FERET database comparing

\begin{tabular}{|c|c|c|c|c|c|c|c|c|}
\hline \multirow[t]{2}{*}{ Dataset size } & \multirow{2}{*}{$\begin{array}{l}\text { Training } \\
\text { images }\end{array}$} & \multirow[t]{2}{*}{ Testing images } & \multicolumn{2}{|l|}{ RIDGE } & \multicolumn{2}{|l|}{ LASSO } & \multicolumn{2}{|l|}{ Proposed } \\
\hline & & & Accuracy & Time (s) & Accuracy & Time (s) & Accuracy & Time (s) \\
\hline 170 & 130 & 40 & 92.4 & 0.59 & 94.6 & 0.6 & 99.7 & 0.48 \\
\hline 280 & 130 & 190 & 91.7 & 0.59 & 94.6 & 0.6 & 98.9 & 0.48 \\
\hline 350 & 130 & 220 & 91.7 & 0.59 & 92.7 & 0.6 & 98.6 & 0.48 \\
\hline 500 & 130 & 370 & 90.6 & 0.59 & 91.5 & 0.6 & 97.6 & 0.48 \\
\hline 750 & 130 & 620 & 90.3 & 0.59 & 89.4 & 0.6 & 96.7 & 0.48 \\
\hline
\end{tabular}

Table 4 Accuracy for different classifiers worked on FERET database

\begin{tabular}{lllllll}
\hline Technique & Dictionary [11] & SVM-MSIFT & GSVM-MSIFT & LASSO & RIDGE & Proposed \\
\hline Accuracy & 93.86 & 92.45 & 94.86 & 94.23 & 92.46 & $\mathbf{9 7 . 1}$ \\
\hline
\end{tabular}

Table 5 Accuracy for different classifiers worked on FGNET database

\begin{tabular}{lllllll}
\hline Technique & Dictionary [11] & SVM-MSIFT & GSVM-MSIFT & LASSO & RIDGE & Proposed \\
\hline Accuracy & - & 86.45 & 91.69 & 93.4 & 92.46 & $\mathbf{9 3 . 3}$ \\
\hline
\end{tabular}

Table 6 Accuracy for different classifiers worked on ORL database

\begin{tabular}{lllllll}
\hline Technique & Dictionary [11] & SVM-MSIFT & GSVM-MSIFT & LASSO & RIDGE & Proposed \\
\hline Accuracy & - & 92.76 & 92.86 & 91.23 & 94.46 & $\mathbf{9 4 . 8}$ \\
\hline
\end{tabular}

Table 7 Accuracy for different classifiers worked on PRIVATE database

\begin{tabular}{lllllll}
\hline Technique & Dictionary [11] & SVM-MSIFT & GSVM-MSIFT & LASSO & RIDGE & Proposed \\
\hline Accuracy & - & 92.59 & 94.86 & 94.63 & 93.46 & $\mathbf{9 8 . 2}$ \\
\hline
\end{tabular}

Table 8 Accuracy for different classifiers worked on FERET database

\begin{tabular}{lllllll}
\hline Technique & Dictionary [11] & SVM-MSIFT & GSVM-MSIFT & LASSO & RIDGE & Proposed \\
\hline Accuracy & - & 92.45 & 94.86 & 94.23 & 92.46 & $\mathbf{9 7 . 4}$ \\
\hline
\end{tabular}

Table 9 Accuracy for different classifiers worked on different databases in combination with MIO-TCD DATABASE

\begin{tabular}{llllll}
\hline $\begin{array}{l}\text { Algorithms in combination } \\
\text { with MIO-TCD database }\end{array}$ & FGNET & FERET & ORL & LFW & PRIVATE \\
\hline SVM-GAUSSIAN & 83.52 & 89.64 & 83.78 & 84.59 & 76.21 \\
LASSO REGRESSION & 88.67 & 91.55 & 87.49 & 83.98 & 84.64 \\
ELASTIC NET LASSO & 92.54 & 93.23 & 94.06 & 93.56 & 93.46 \\
PROPOSSED (LRGS) & 95.65 & 94.60 & 96.23 & 95.54 & 98.86 \\
\hline
\end{tabular}




\section{LRGS}

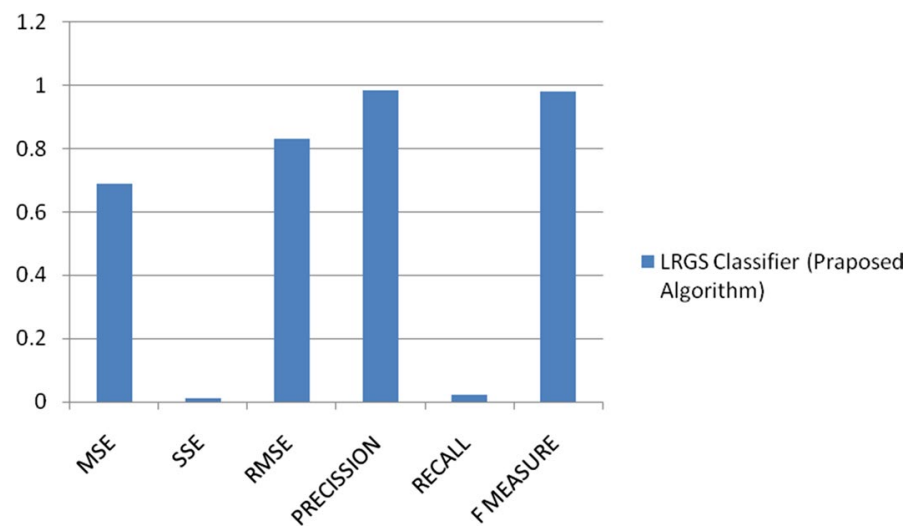

Fig. 8 Different mertics for Proposed (LRGS) classifiers worked on different databases in combination with MIO-TCD DATABASE

Figure 8 explains, the performance metrics of our proposed algorithm (LRGS) worked on different databases in combination with MIO-TCD DATABASE; precision is $98.3 \%$, recall is $0.022 \%$, MSE is 0.69 , SSE is 0.011 , RMSE is 0.83 and F-measure is 98.2.

\section{Conclusion}

In this paper, 5 different databases (combined with MIO-TCD vehicle Database) are tested with different algorithms and some techniques are compared with respect to references. In results section of this paper, 3 different approaches are compared with multivariant mode. At most of the references are authors implemented FERET databases, to show case the best outcome cited the comparison this dataset results are compared with other results are compared at dataset level. The proposed approach results in best accuracy with less time of execution. In future the enhanced process can be applied on video processing units for real time operations of detection in objects. All these accuracies obtained with respect to the number of test samples with subjective mean of all classified accuracies.

\footnotetext{
Abbreviations

SIFT: Scale Invariant Feature Transform; RR: Ridge regression; EN: Elastic net; LR: Lasso regression; LRGS: Lasso regression with Gaussian Support Vector Msachines; SVM: Support Vector Machine; MSIFT: Multi Scale Invariant Feature Transform; ARD: Automatic Relevance Detection; GSVM: Gaussian Support Vector Machine; MSVM: Multi Support Vector Machine; LASSO: Least Absolute Shrinkage and Selection Operator; PCA: Principle Component Analysis; HOG: Histogram of oriented gradients; GLM: Generalised linear model; MSE: Mean Square Error; SSE: Sum of Squared Error; RMSE: Root Mean Square Error; ORL: Olivetti Research Laboratory; FERET: Facial Recognition Technology; LFW: Labeled Faces in the Wild; FG-NET: The face and gesture recognition network; NBC: Naive bayes Classifier; RFC: Random Forest Classifier; FGSVM: Fuzzy C Means Gaussian SVM.
}

Acknowledgements

Not applicable.

Authors' contributions

Not applicable.

Funding

Not applicable.

Availability of data and materials

Not applicable. 


\section{Competing interests}

The authors declare that they have no competing interests.

\section{Author details}

${ }^{1}$ Computer Science, Krishna University, Machilipatnam, A.P, India. ${ }^{2}$ Department of IT, Wollega University, Nekemte, T.R, Ethiopia.

Received: 9 March 2020 Accepted: 1 November 2020

Published online: 11 November 2020

\section{References}

1. Nguyen DT, Park KR. Enhanced gender recognition system using an improved histogram of oriented gradient (HOG) feature from quality assessment of visible light and thermal images of the human body. Sensors. 2016;16(7):1134.

2. Tianyu L, Fei L, Rui W. Human face gender identification system based on MB-LBP. In: The 30th Chinese Control And Decision Conference (2018 CCDC). IEEE Publisher, Shenyang, China; 2018, p. 1721-5. https://doi.org/10.1109/ ccdc.2018.8407405.

3. Cerkezi L, Topa C. Gender recognition with uniform local binary patterns. In: 26 th signal processing and communications applications conference (SIU), IEEE publisher, Izmir; 2018, p. 1-4. https://doi.org/10.1109/siu.2018.8404587.

4. Bianconi F, Smeraldi F, Abdollahyan M, Xiao P. On the use of skin texture features for gender recognition: an experimental evaluation. In: 2016 sixth international conference on image processing theory, tools and applications (IPTA), IEEE publisher, Oulu; 2016, p. 1-6. https://doi.org/10.1109/ipta.2016.7821018.

5. Zhou B, Lapedriza A, Khosla A, Oliva A, Torralba A. Places: a 10 million image database for scene recognition. IEEE Trans Pattern Anal Mach Intell. 2017;40(6):1452-64.

6. Zeni LF, Jung C. Real-time gender detection in the wild using deep neural networks. In: 31st SIBGRAPI conference on graphics, patterns and images (SIBGRAPI), IEEE publisher, Parana, Brazil; 2018, p. 118-25. https://doi.org/10.1109/ sibgrapi.2018.00022.

7. Redmon J, Farhadi A. Yolo9000: better faster stronger. In: The IEEE conference on computer vision and pattern recognition (CVPR), IEEE Computer Society; 2017, p. 6517-25. https://doi.org/10.1109/cvpr.2017.690.

8. Liu W, Anquelov D, Erhan D, Szegedy C, Reed S, Fu CY, Berg AC. Berg. SSD: Single shot multibox detector. In: Lecture notes in computer science, Springer publisher, ECCV 2016, Part I, LNCS 9905; 2016, p. 21-37. https://doi. org/10.1007/978-3-319-46448-0_2.

9. Levi G, Hassner T. Age and gender classification using convolutional neural networks. In: IEEE workshop on analysis and modeling of faces and gestures (AMFG), at the IEEE Conf. on Computer Vision and Pattern Recognition (CVPR), Boston; 2015, p. 34-42.

10. Ranjan R, Pate VM, Chellappa R. Hyperface: a deep multitask learning framework for face detection landmark localization pose estimation and gender recognition. IEEE Trans Pattern Anal Mach Intell. 2016;10(10):1-16.

11. Mahendran A, Vedaldi A. Visualizing deep convolutional neural networks using natural pre-images. Int J Comput Vis. 2016;120(3):233-55.

12. Kishore-Galla DK, Babu-Reddy M. Gender classification based on similarity features through SURF and SVM. Int J Knowl Eng Data Mining. 2019;6(1):89-104. https://doi.org/10.1504/ijkedm.2019.097353.

13. Chen C, Ross A. A multi-task convolutional neural network for joint iris detection and presentation attack detection. In: 2018 IEEE Winter applications of computer vision workshops (WACVW), IEEE publisher; 2018, p. 44-51. https:// doi.org/10.1109/wacrw.2018.00011.

14. Zhou B, Khosla A, Lapedriza A, Oliva A, Torralba A. Learning deep features for discriminative localization. In: 2016 IEEE conference on computer vision and pattern recognition, IEEE Computer Society; 2016, p. 2921-9. https://doi. org/10.1109/cvpr.2016.319.

15. Wang J, Xu C, Yang X, Zurada JM. A novel pruning algorithm for smoothing feedforward neural networks based on group lasso method. IEEE Trans Neural Netw Learn Syst. 2018;29(5):2012-24.

16. Montazzolli S, Jung C. Real-time Brazilian license plate detection and recognition using deep convolutional neural networks. In: 30th SIBGRAPI conference on graphics, patterns and images, IEEE Publisher; 2017, p. 55-62. https://doi. org/10.1109/sibgrapi.

17. Kishore GDK, Babu Reddy M. Detecting human and classification of gender using facial images MSIFT features based GSVM. Int J Recent Technol Eng. 2019;8(3):1466-71.

18. Galla DK, Mukamalla B, Chegireddy RP. Classification of multi-label object based on MSIFT feature probabilistic fuzzy c-means clustering classified by GSVM. J Mech Continua Math Sci. 2020;15(4):247-63.

19. Vinay A, Shekhar VS, Balasubramanya-Murthy KN, Natarajan S. Face recognition using gabor wavelet features with PCA and KPCA—a comparative study. Procedia Comput Sci. 2015;57:650-9.

20. Kishore GDK, Babu Reddy M. Analysis and prototype sequences of face recognition techniques in real-time picture processing. In: 6th international conference on FICTA, intelligent engineering informatics, advances in intelligent systems and computing; 2018, Vol. 695, p. 323-335. https://doi.org/10.1007/978-981-10-7566-7.

\section{Publisher's Note}

Springer Nature remains neutral with regard to jurisdictional claims in published maps and institutional affiliations. 